\title{
Relative survival in early-stage cancers in the Netherlands: a population-based study
}

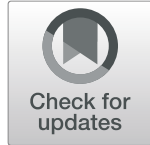

\author{
Avinash G. Dinmohamed ${ }^{1,2,3^{*}}$ (D, Valery E. P. P. Lemmens ${ }^{1,2}$, Ignace H. J. T. de Hingh ${ }^{1,4,5}$ and Otto Visser ${ }^{6}$
}

\begin{abstract}
In this nationwide, population-based study, we assessed 10-year relative survival among 225,305 patients with ten early-stage cancers diagnosed in the Netherlands during 2004-2015. This study aimed to ascertain which early-stage cancer is associated with minimal or no excess mortality and likely to be diagnosed in individuals who are otherwise more healthy or health-conscious than their counterparts in the general population. Tenyear relative survival marginally exceeded $100 \%$ in patients with early-stage prostate cancer, while it was close to $100 \%$ for patients with ductal carcinoma in situ (DCIS) and stage I cancers of the breast, skin (melanoma), testis, and thyroid. In contrast, patients with early-stage oral/pharyngeal, bladder, lung, and pancreatic cancers experienced considerable excess mortality, reflected by a 10-year relative survival of $74.9 \%, 69.4 \%, 45.5 \%$, and $33.9 \%$, respectively. Collectively, the life expectancy of patients with DCIS and early-stage cancers of the prostate, breast, skin (melanoma), testis, and thyroid parallels the expected survival of an age-, sex-, and calendar year-matched group from the general population. Our study findings add to the controversy surrounding overdiagnosis of particular early-stage cancers that are generally not destined to metastasis or cause excess mortality.
\end{abstract}

Keywords: Cancer, Relative survival, Early-stage, Epidemiology, Registry, Population-based

To the Editor,

Recently, Marcadis et al. performed a comprehensive analysis of relative survival in 281,970 patients with ten early-stage cancers using data from the Surveillance, Epidemiology, and End Results Program - a study that was hitherto lacking in contemporary literature [1]. They identified five early-stage cancers-i.e., ductal carcinoma in situ (DCIS) and early-stage cancers of the prostate, skin (melanoma), thyroid, and breast-with 10-year relative survival exceeding $100 \%$, suggesting that patients with these early-stage cancers have greater longevity compared to an equivalent group from the general population. This finding favors the premise that these early-stage cancers-which presumably lack metastatic potential and have limited lethal consequences-are generally detected incidentally or via screening in individuals who are otherwise more healthy or health-conscious than their counterparts in the general population [2].

\footnotetext{
* Correspondence: a.dinmohamed@iknl.nl

${ }^{1}$ Department of Research and Development, Netherlands Comprehensive

Cancer Organisation (IKNL), Godebaldkwartier 419, 3511 DT Utrecht, The

Netherlands

2Department of Public Health, Erasmus MC, University Medical Center

Rotterdam, Rotterdam, The Netherlands

Full list of author information is available at the end of the article
}

(c) The Author(s). 2020 Open Access This article is licensed under a Creative Commons Attribution 4.0 International License, which permits use, sharing, adaptation, distribution and reproduction in any medium or format, as long as you give appropriate credit to the original author(s) and the source, provide a link to the Creative Commons licence, and indicate if changes were made. The images or other third party material in this article are included in the article's Creative Commons licence, unless indicated otherwise in a credit line to the material. If material is not included in the article's Creative Commons licence and your intended use is not permitted by statutory regulation or exceeds the permitted use, you will need to obtain permission directly from the copyright holder. To view a copy of this licence, visit http://creativecommons.org/licenses/by/4.0/. The Creative Commons Public Domain Dedication waiver (http://creativecommons.org/publicdomain/zero/1.0/) applies to the data made available in this article, unless otherwise stated in a credit line to the data. 
This phenomenon can to some extent be referred to as overdiagnosis [3].

At present, it is unknown whether the findings of Marcadis et al. extend to populations outside the USA. Therefore, we sought to complement their findings by delineating the relative survival of patients with early-stage cancers in the Netherlands.

We selected patients with ten early-stage cancers diagnosed during 2004-2015 from the nationwide Netherlands Cancer Registry (NCR). The selection of early-stage cancers and the study period is congruent with the selection as per Marcadis et al. [1]. The Privacy Review Board of the NCR approved the use of anonymous data for this study.

The primary endpoint was 10-year relative survival, defined as the time from diagnosis until death, emigration, or end of follow-up (February 1, 2019), whichever occurred first. Relative survival was calculated according to the cohort methodology to estimate disease-specific survival [4]. Relative survival is the ratio of the observed survival of patients to the expected survival of an age-, sex-, and calendar yearmatched group from the general population. Expected survival was calculated as per the Ederer II methodology using Dutch life tables, stratified by age, sex, and calendar year. All analyses were performed using STATA (version 14.2, StataCorp).

Our analytical cohort included 225,305 patients with ten early-stage cancers diagnosed in the Netherlands during 2004-2015 (Fig. 1). Ten-year relative survival marginally exceeded $100 \%$ in patients with early-stage prostate cancer (101.4\%; 95\% CI, 100.7-102.0\%), while it was close to $100 \%$ for patients with DCIS and stage I cancers of the skin (melanoma), testis, and thyroid (Fig. 2). Patients with stage I breast cancer experienced minimal excess mortality, while patients with early-stage oral/pharyngeal, bladder, lung, and pancreatic cancers experienced considerable excess mortality (Fig. 2).

In this nationwide, population-based study, the life expectancy of patients with early-stage prostate cancer slightly surpassed that of an equivalent group from the general population. This finding is congruent-albeit to a lesser extent-with those observed in the USA (Fig. 2) [1]. Besides, relative survival exceeded $100 \%$ among US patients with DCIS and early-stage cancers of the thyroid, skin (melanoma), and breast. In the Netherlands, however, relative survival in these patients ranges between $96 \%$ and 100\%, which closely mirrors the disease-specific survival in the USA [1] Hence, relative survival in these early-stage cancers is likely to be overestimated in the USA. Seemingly, there is an overrepresentation of health-conscious individuals (e.g., those from higher socioeconomic

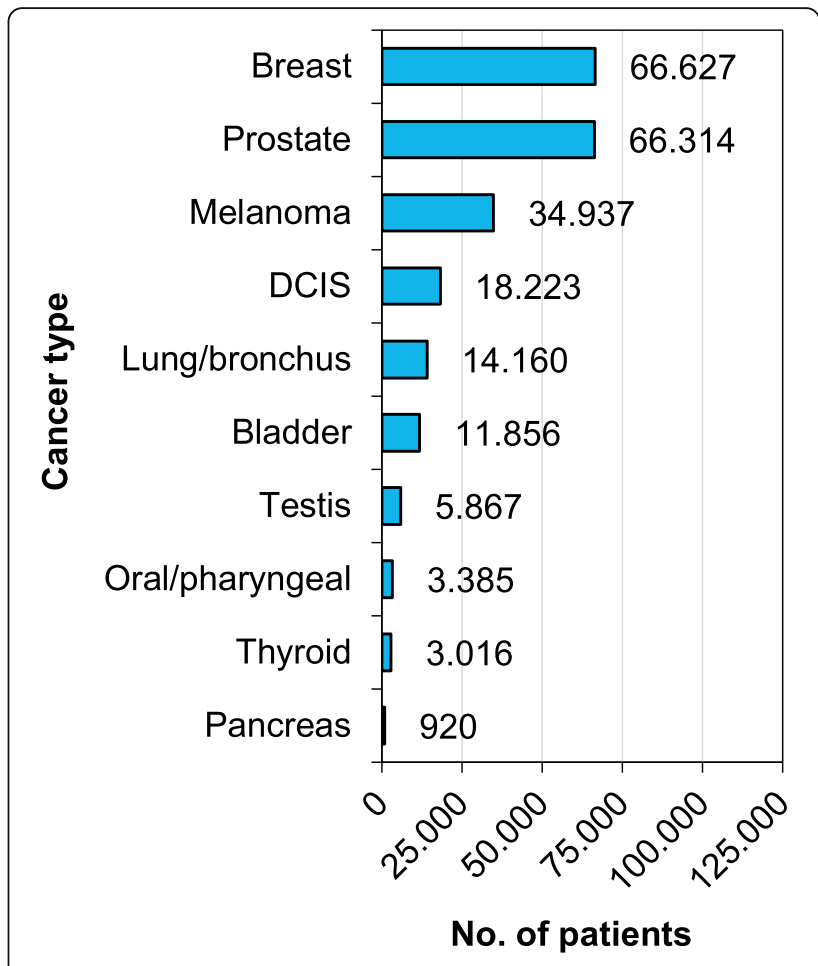

Fig. 1. Distribution of early-stage cancers in the Netherlands

groups or with adequate insurance coverage) in the US patient population with early-stage cancers. Moreover, these patients have a higher life expectancy than their counterparts in the general population and perhaps more diligently participate in periodic health examinations and cancer screening programs (i.e., healthy-user bias) - all of which may render a cancer diagnosis earlier in the disease course (i.e., lead-time bias) $[2,5,6]$. Sex- and treatment-related factors might also be at play with regard to disparities in relative survival between the Netherlands and the USA. The effect of sex and variation in treatment on relative survival could not be evaluated since information on sex and therapy was not analyzed in the study by Marcadis et al. [1].

Collectively, it can be reasoned that the magnitude of potential cancer overdiagnosis and health inequalities regarding access to screening, diagnosis, treatment, and follow-up services is less extensive in the Netherlands than in the USA, because all Dutch residents have equal access to health care services-regardless of their socioeconomic position and race, ethnicity, and gender. This topic provides an avenue for future research to close the gap in cancer survival between the Netherlands and the USA. This research requires detailed analyses on the drivers of survival 


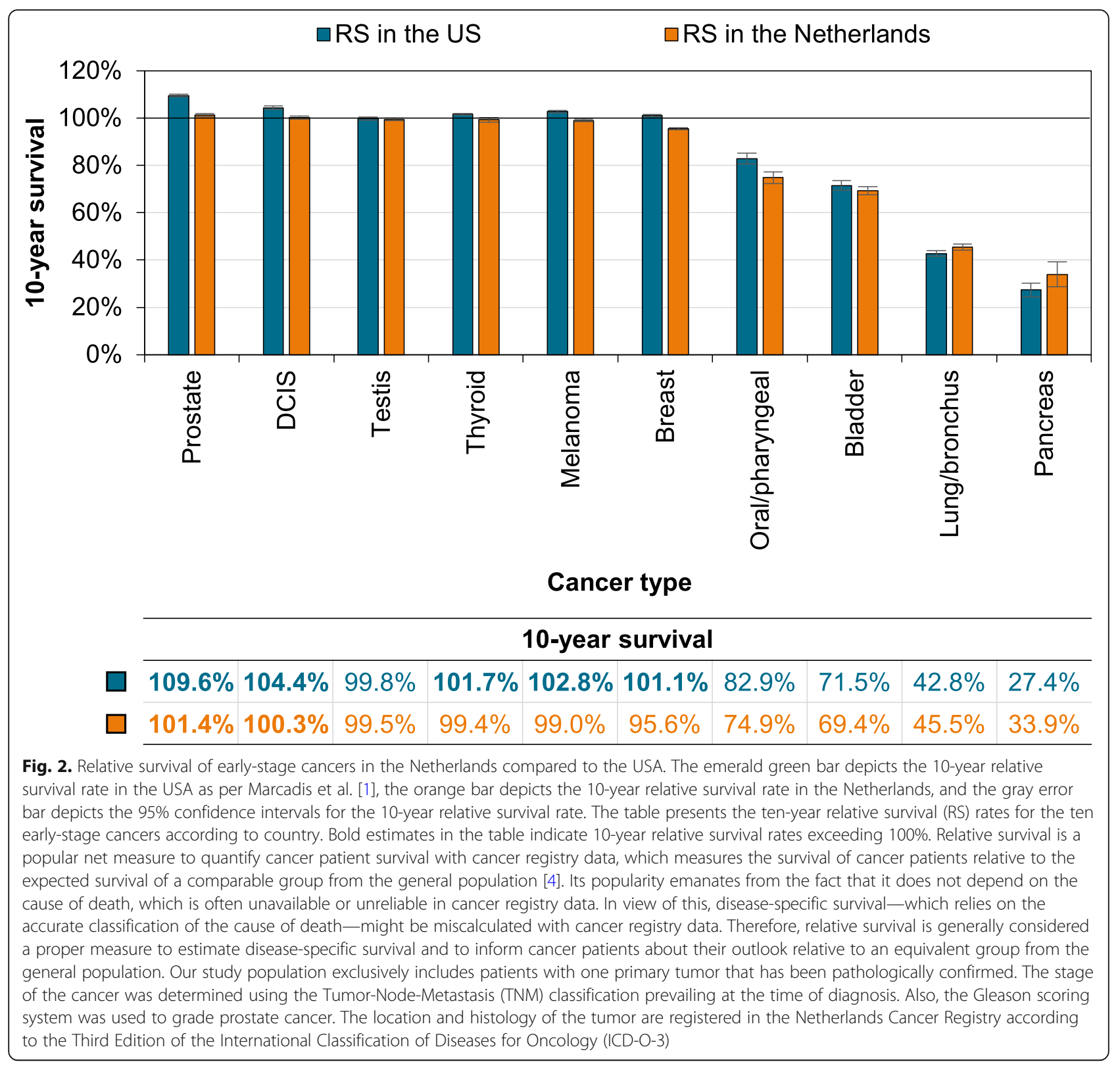

disparities in early-stage cancers between the Funding

Netherlands and the USA. None.

\section{Abbreviations}

Cl: Confidence interval; DCIS: Ductal carcinoma in situ; US: United States; NCR: Netherlands Cancer Registry

\section{Acknowledgements}

The authors would like to thank the registration clerks of the Netherlands Cancer Registry (NCR) for their dedicated data collection. The nationwide population-based NCR is maintained and hosted by the Netherlands Comprehensive Cancer Organisation (IKNL).

\section{Authors' contributions}

AGD designed the study. AGD and OV analyzed the data. OV collected the data. AGD wrote the manuscript with contributions from all authors, who also interpreted the data, and read, commented on, and approved the final version of the manuscript.

\section{Availability of data and materials}

The data that support the findings of this study are available via The Netherlands Comprehensive Cancer Organisation. These data are not publicly available and restrictions apply to the availability of the data used for the current study. However, these data are available upon reasonable request and with permission of The Netherlands Comprehensive Cancer Organisation.

\section{Ethics approval and consent to participate}

According to the Central Committee on Research involving Human Subjects (CCMO), this type of observational study does not require approval from an ethics committee in the Netherlands. The Privacy Review Board of the Netherlands Cancer Registry approved the use of anonymous data for this study. 


\section{Consent for publication}

Not applicable.

\section{Competing interests}

None.

\section{Author details}

'Department of Research and Development, Netherlands Comprehensive Cancer Organisation (IKNL), Godebaldkwartier 419, 3511 DT Utrecht, The Netherlands. ${ }^{2}$ Department of Public Health, Erasmus MC, University Medical Center Rotterdam, Rotterdam, The Netherlands. ${ }^{3}$ Department of Hematology, Amsterdam UMC, Cancer Center Amsterdam, Amsterdam, The Netherlands. ${ }^{4}$ Department of Surgical Oncology, Catharina Hospital, Eindhoven, The Netherlands. ${ }^{5} \mathrm{GROW}$-School for Oncology and Developmental Biology, Maastricht University, Maastricht, The Netherlands. ${ }^{6}$ Department of Registration, Netherlands Comprehensive Cancer Organisation (IKNL), Utrecht, The Netherlands.

Received: 6 March 2020 Accepted: 1 May 2020

Published online: 12 May 2020

\section{References}

1. Marcadis AR, Marti JL, Ehdaie B, et al. Characterizing Relative and DiseaseSpecific Survival in Early-Stage Cancers. JAMA Intern Med. 2019.

2. Welch HG, Kramer BS, Black WC. Epidemiologic Signatures in Cancer. N Engl J Med. 2019:381(14):1378-86.

3. Welch HG, Black WC. Overdiagnosis in cancer. J Natl Cancer Inst. 2010; 102(9):605-13.

4. Dickman PW, Adami HO. Interpreting trends in cancer patient survival. J Intern Med. 2006;260(2):103-17.

5. Shrank WH, Patrick AR, Brookhart MA. Healthy user and related biases in observational studies of preventive interventions: a primer for physicians. J Gen Intern Med. 2011;26(5):546-50.

6. Walker GV, Grant SR, Guadagnolo BA, et al. Disparities in stage at diagnosis, treatment, and survival in nonelderly adult patients with cancer according to insurance status. J Clin Oncol. 2014;32(28):3118-25.

\section{Publisher's Note}

Springer Nature remains neutral with regard to jurisdictional claims in published maps and institutional affiliations. 